\title{
The Relative Expansion of the Universe
}

\author{
Lee Qing Jong \\ Free-lancer, Kuala Lumpur, Malaysia
}

Email address:

whosyourdaddy913@hotmail.com

\section{To cite this article:}

Lee Qing Jong. The Relative Expansion of the Universe. American Journal of Astronomy and Astrophysics. Vol. 3, No. 3, 2015 , pp. 37-39. doi: 10.11648/j.ajaa.20150303.11

\begin{abstract}
This is an alternate explanation to the redshift effect and increasing distance between galaxies. Everything in the universe excluding empty space is shrinking, including elementary particles. In contrast to everything else, the empty space would appear to be expanding. This idea is compatible with most of established science, such as the theory of relativity, the doppler effect, the big rip idea. And, it also helps explain dark energy. An idea that is not very well understood yet.
\end{abstract}

Keywords: Universe, Expansion, Cosmology, Physics, Relativity

\section{Introduction}

The expansion of the universe has been widely accepted by the scientific community, but there is a paradoxical problem with the expanding universe idea. When we look into a telescope, we can see that the distance between galaxies is increasing and we can tell by the redshift effect that everything is receding away from us.

The problem lies in the idea that the universe is increasing in volume, which is simply unsatisfying. Since the universe is the sum of everything within reality, the idea of it expanding is nonsensical. An alternative explanation would be to assume that everything in the universe excluding empty space is decreasing in volume, and the rate at which it is shrinking is increasing. This includes elementary particles. If we assume this is the case, we would be able to explain the redshift and increasing distance between galaxies without the paradox of the universe increasing in volume.

\section{Compatibility with Established Science}

\subsection{Theory of Relativity}

Einstein's theory of relativity suggests that object A moving away from object B is the same as object B moving away from object A. If the surface area of everything is decreasing, it would be indistinguishable from actually moving farther apart in space. We are unable to detect these changes because our forms of measurement shrink at the same rate as everything else. ${ }^{[1]}$

\subsection{Doppler Effect}

Since the surface area of two objects decreasing is equivalent to them moving farther apart, the redshift effect can be observed. Therefore, the shrinking particle idea is as compatible with the Doppler effect as the expanding universe idea. $^{[2][3]}$

\subsection{The Big Rip}

Similar to the expanding universe idea, the shrinking idea would also suggest increasing distance between objects will eventually cause all interaction between particles impossible. ${ }^{[4]}$

\subsection{Everything Else}

One of the main attributes of this idea, is its compatibility with established science. It is as compatible with established science as the expanding universe idea, it does not contradict anything the expanding universe idea does not also contradict.

\section{Dark Energy}

Originally, scientists thought the expansion of the universe was slowing down. But upon further investigation, they discovered that this was not the case. They found that the expansion was actually accelerating. From what we understood about gravity, this should not have been the case. Scientists tried to explain this phenomenon by assuming there is an undetectable force acting on them called Dark Energy. But if we assume that the universe is not expanding. And it is the elementary particles that are shrinking instead, we can 
explain why the rate at which galaxies are moving apart appears to be accelerating. ${ }^{[5][6]}$

\subsection{Newton's Laws of Motion}

Newton's first law of motion states that an object will remain at rest or continue moving at a constant velocity, unless acted upon by an external force. Similar to how two objects fall toward each other at an accelerating rate, if unobstructed. The same law would also apply to whatever makes up elementary particles. ${ }^{[7][8]}$

\subsection{Concept of Infinity}

It is important to understand that infinity not only extends outward, but also inward. What I mean is that there does not seem to be a limit to how small something can be. One can never know if they have found the smallest possible "thing" in existence, until something smaller has been found. Before the electron was discovered in 1895 , we had no idea that there was something smaller than an atom. ${ }^{[9]}$ Because of the nature of infinity, arguing about whether or not there is a limit to how small something can get and whether or not there is something smaller than elementary particles is pointless. For the sake of productivity, assume that there is an infinite number of "things" smaller than our current smallest object. The elementary particles. And assume that all of those infinitely small "things" all follow the same law of being attracted to one another. Under these assumptions, if whatever makes up elementary particles were to shrink. So would the elementary particles. This is the same for whatever makes up elementary particles. If the building blocks of whatever elementary particles are composed of were to shrink, so would the building blocks of elementary particles. And so on, and so on. ${ }^{[10][11][12][13]}$

\subsection{Acceleration}

If these infinitely small "things" were to shrink, the rate at which they shrink would increase over time. Because an object will remain at rest or continue moving at a constant velocity unless acted upon by an external force, that force being the assumed "shrinking force" that causes the infinitely small "things" to shrink. And without another external force to counteract this "shrinking force", the rate of shrinking will eventually get so fast that gravity will no longer be able to compensate for it and keep galaxies in their relative positions. ${ }^{[14][15][16]}$

\section{Why is Redshift not Observed on Earth}

If everything is shrinking, one would expect everything we observe to show signs of redshift. The reason this is not the case is because when we look at distant galaxies, we know those galaxies are made of smaller parts. However, we obviously lack the technology to observe the atoms that make up those galaxies, so we consider the entire galaxy as one thing. If we were instantly teleport to those distant galaxies, we would most likely detect no signs of redshift. This is because of the difference in size between us and those galaxies.
When we look at those distant galaxies, even though they are traveling at a high speed through space. We can hardly see any movement from them. Similarly, from the subatomic perspective we would appear to move rather sluggishly. This is because distance is relative to the size of the object. If an object were small enough, one centimeter would feel a lightyear. Even though the actual length of a lightyear is still more than one centimeter, the amount of distance the object needs to cover would feel like lightyear. And since an atom is so small, even though the rate of shrinking is high, the distance it covers is rather miniscule. The smaller something is, the closer the object is to it's point of origin. And the closer an object is to it's point of origin, the more relative distance it needs to cover. As the size decreases, so does the rate of shrinking decrease from our perspective. And since the rate of recession is so insignificant, no redshift can be detected. The rate of shrinking would probably look something like this. $^{[17][18][19]}$

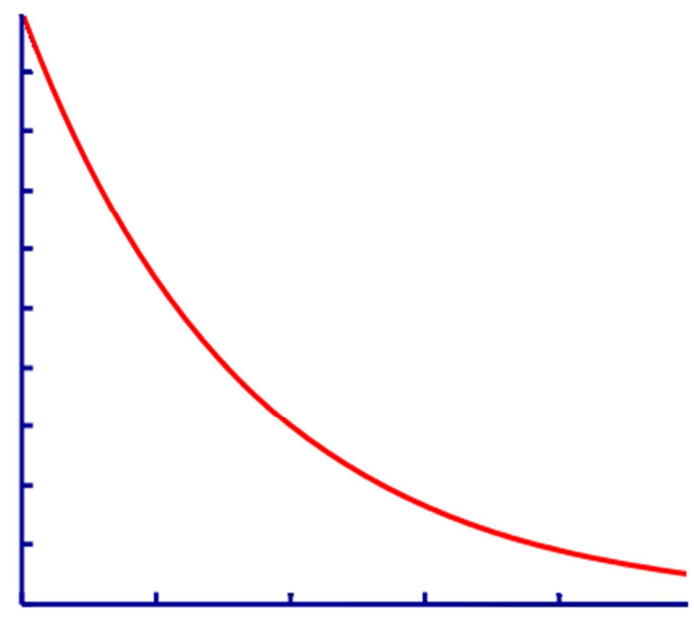

Figure 1. Relationship between rate of shrinking and size of object.

$\mathrm{x}=$ rate of shrinking $\mathrm{y}=$ size of object

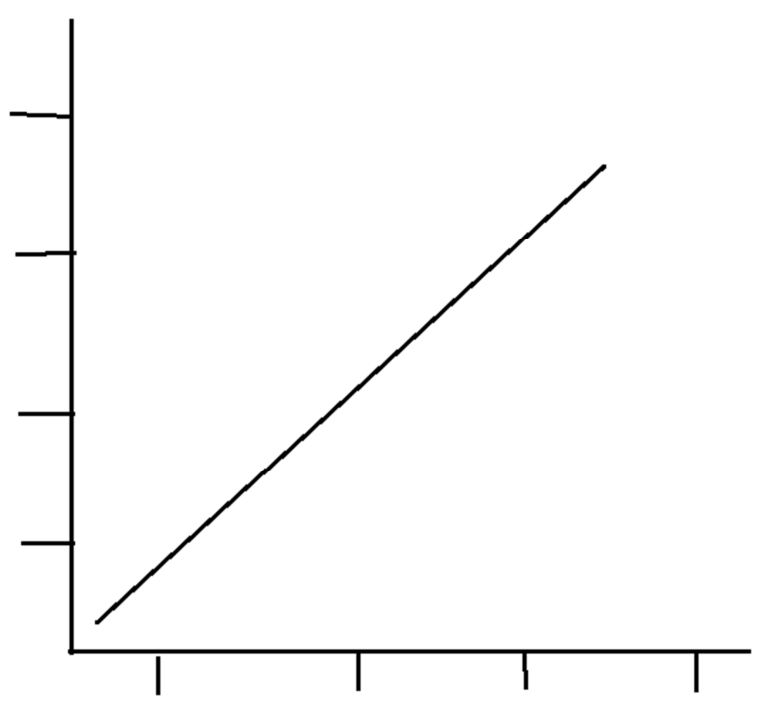

Figure 2. Relationship between rate of shrinking and size of object. $\mathrm{x}=$ rate of shrinking $\mathrm{y}=$ size of object 


\section{Practical Uses}

It provides an explanation to dark energy, a phenomenon that is not well understood. It removes the unsatisfying idea of the universe speeding up as time passes, akin to throwing a ball upward and watching it fly up faster and faster.

\section{References}

[1] Wald, Robert M. (1984), General Relativity, University of Chicago Press, ISBN 0-226-87033-2.

[2] "Doppler and the Doppler effect", E. N. da C. Andrade, Endeavour Vol. XVIII No. 69, January 1959 (published by ICI London). Historical account of Doppler's original paper and subsequent developments

[3] Evans, D. H.; McDicken, W. N. (2000). Doppler Ultrasound (Second ed.). New York: John Wiley and Sons. ISBN 0-471-97001-8.

[4] Caldwell, Robert R.; Kamionkowski, Marc and Weinberg, Nevin N. (2003). "Phantom Energy and Cosmic Doomsday". Physical Review Letters 91 (7): 071301. arXiv: astro-ph/0302506. Bibcode: 2003PhRvL..91g1301C. doi:10.1103/PhysRevLett.91.071301.PMID 12935004.

[5] Peebles, P. J. E. and Ratra, Bharat (2003). "The cosmological constant and dark energy". Reviews of Modern Physics 75 (2): 559-606. arXiv: astro-ph/0207347. Bibcode: 2003RvMP...75..559P. doi:10.1103/RevModPhys.75.559.

[6] Riess, Adam G.; Filippenko; Challis; Clocchiatti; Diercks; Garnavich; Gilliland; Hogan; Jha; Kirshner; Leibundgut; Phillips; Reiss; Schmidt; Schommer; Smith; Spyromilio; Stubbs; Suntzeff; Tonry (1998). "Observational evidence from supernovae for an accelerating universe and a cosmological constant". Astronomical J. 116 (3): 1009-38. arXiv: astro-ph/9805201.

[7] Galili, I.; Tseitlin, M. (2003). "Newton's First Law: Text, Translations, Interpretations and Physics Education". Science \& Education 12 (1): 45-73. Bibcode: 2003Sc\&Ed..12...45G. doi:10.1023/A:1022632600805.
[8] Raymond A. Serway; Jerry S. Faughn (2006). College Physics. Pacific Grove CA: Thompson-Brooks/Cole. p. 161.ISBN 0-534-99724-4.

[9] Andrew G. van Melsen (1952). From Atomos to Atom. Mineola, N.Y.: Dover Publications. ISBN 0-486-49584-1.

[10] Wolff, P. Breakthroughs in Mathematics, 1963, New York: New American Library, pp 47-8

[11] Karttunen, Lauri (1974) [1]. Theoretical Linguistics 1 181-94. Also in Pragmatics: A Reader, Steven Davis (ed.), pages 406-415, Oxford University Press, 1991.

[12] Garay, Luis J. (January 1995). "Quantum gravity and minimum length". International Journal of Modern Physics A 10 (2): 145 ff. arXiv:gr-qc/9403008v2. Bibcode: 1995IJMPA..10..145G. doi:10.1142/S0217751X95000085.

[13] John Baez, The Planck Length

[14] Hannah, J, Hillier, M J, Applied Mechanics, p221, Pitman Paperbacks, 1971

[15] J.R. Forshaw, A.G. Smith (2009). Dynamics and Relativity. Wiley. p. 247. ISBN 978-0-470-01460-8.

[16] Das, A. (1993) The Special Theory of Relativity, A Mathematical Exposition, Springer, ISBN 0387940421.

[17] Lee, John M. (2013), Axiomatic Geometry, Pure and Applied Undergraduate Texts 21, American Mathematical Society, p. 134, ISBN 9780821884782.

[18] Leike, A. (2002). "Demonstration of the exponential decay law using beer froth". European Journal of Physics 23: 21. Bibcode: 2002EJPh...23...21L. doi:10.1088/0143-0807/23/1/304.

[19] Swirski, Peter. Of Literature and Knowledge: Explorations in Narrative Thought Experiments, Evolution, and Game Theory. New York: Routledge. ISBN 0-415-42060-1. 